\title{
Fixation point offsets facilitate endogenous saccades
}

\author{
RICHARD A. ABRAMS, HEATHER M. OONK, and JAY PRATT \\ Washington University, St. Louis, Missouri
}

\begin{abstract}
Subjects produced saccades to continuously visible targets that were signaled by the pitch, not the location, of an auditory signal. Such endogenous saccades were initiated more quickly when the visual fixation point disappeared $200 \mathrm{msec}$ before the signal (thus producing a "gap"), even though the alerting benefits of such a warning were eliminated by an earlier warning tone. The presence of the gap effect under these circumstances shows that the effect is more general than was previously believed: Visual fixation point offsets facilitate saccades by affecting oculomotor processes related to both visually elicited (exogenous) and centrally produced (endogenous) saccades. In addition, the magnitude of the gap effect for endogenous saccades was significantly smaller than that for exogenous saccades, suggesting that at least some of the effect arises in relatively early processes, such as those involved in the processing of sensory signals, and not exclusively in later processes, such as those involved in the preparation and production of saccades.
\end{abstract}

Because saccadic eye movements play such an important role in a person's ability to construct a coherent representation of his or her environment, researchers have extensively studied many aspects of them. There has recently been renewed interest in an effect first reported by Saslow (1967). He found that saccades from a fixation point to a suddenly appearing target were initiated more quickly if the fixation point disappeared shortly before the appearance of the target. Fischer and colleagues (e.g., Fischer \& Ramsperger, 1984) have suggested that these short-latency saccades, which they call express saccades, are unique, and that they come from a class of movements distinct from regular (i.e., longer latency) saccades. In support of this claim, they have reported the presence of bimodal latency distributions in many subjects (Fischer $\&$ Weber, 1993). The faster mode of the latency distribution presumably includes express saccades, and the slower mode, the regular saccades. Yet although it is quite easy to show that advance offset of fixation reduces saccade latencies, many researchers have been unable to obtain bimodal latency distributions (e.g., Cameron \& Lennie, 1993; Kingstone \& Klein, 1993b; Reuter-Lorenz, Hughes, \& Fendrich, 1991; Wenban-Smith \& Findlay, 1991). The latter researchers have challenged Fischer's characterization and prefer instead to discuss the gap effect, the term gap referring to the time interval between fixation offset and target onset. Such a gap does reliably reduce saccade latencies.

The research reported in this paper was supported by Grant R29MH45145 from the National Institutes of Health. The authors thank Heidi Korthase and Shawn Christ for excellent technical assistance. Correspondence should be addressed to R. A. Abrams, Department of Psychology, Washington University, St. Louis, MO 63130 (e-mail: rabrams@artsci.wustl.edu).

\section{Explanations for the Gap Effect}

Thus, although there is agreement that a gap can reduce saccade latencies, there is not any general agreement regarding the mechanisms that underlie the effect. Fischer and Weber (1993) argue that the effect is attentional. According to them, the offset of fixation permits subjects to disengage their attention in advance of target appearance. During such a disengaged state, the target is presumed to engage attention more easily when it appears, allowing subjects to respond to it more quickly. Such an explanation might predict that any response would be facilitated by a gap. However, only target-directed saccades appear to benefit: If subjects are required to look away from a target when it appears (an antisaccade), the gap has little or no effect (Reuter-Lorenz et al., 1991; Reuter-Lorenz, Oonk, Barnes, \& Hughes, 1995; see also Kingstone \& Klein, 1993a, for additional evidence inconsistent with an attentional explanation of the gap effect). There is also some question about the presence of a gap effect for manual responses: Some researchers have reported the absence of such effects for manual keypress responses (e.g., Reuter-Lorenz et al., 1991; Ross \& Ross, 1981), but more recently, others have found small but reliable effects of a gap on aimed hand movement latencies (Bekkering, Pratt, \& Abrams, 1996). In Bekkering et al.'s study, the gap had a larger effect on eye movement latencies than on hand movement latencies. Thus, at least some of the latency reduction afforded by a gap is due to factors other than enhanced target detection, because otherwise all types of responses would be expected to benefit equally. Instead, the effect appears to arise at least partly from enhanced premotor or motoric processes associated with the generation of saccades to peripheral targets (Reuter-Lorenz et al., 1995). 


\section{Endogenous Versus Exogenous Saccades}

There is, however, one potential limitation common to the previous work on the gap effect. In particular, in all studies to date, exogenous stimuli have been used to signal the target for the saccade and begin the latency interval. ${ }^{1}$ As a result, the saccades produced were stimulus driven, not centrally produced. This is true for studies done with visual stimuli (e.g., Fischer \& Ramsperger, 1984) and auditory stimuli (e.g., Fendrich, Hughes, \& Reuter-Lorenz, 1991), as well as for studies of saccades, antisaccades, and manual responses (e.g., Bekkering et al., 1996; Reuter-Lorenz et al., 1991). As a result, it is not known to what extent a gap may facilitate more centrally controlled or endogenous saccades. The lack of research on the gap effect for endogenous saccades seems especially significant, given that such saccades are those likely to be emitted in the normal course of scanning a fairly stable environment. ${ }^{2}$

Ample evidence suggests that endogenously produced saccades differ from exogenously produced ones, raising the possibility of different gap effects. In particular, different neuroanatomical structures may be responsible for endogenous and exogenous saccades. For example, Guitton, Buchtel, and Douglas (1985) have shown that patients with frontal lobe lesions have difficulty producing endogenous saccades away from visual targets (antisaccades), but that they can easily produce exogenous, visually triggered saccades toward visual targets, suggesting a greater role of the frontal eye fields in endogenous as opposed to exogenous saccades. Evidence from several other sources also suggests the existence of two parallel pathways that are involved in the production of saccades: a phylogenetically more primitive system involved mainly in reflexive, exogenous eye movements; and a more advanced system responsible for inhibiting reflexive saccades and producing endogenous, voluntary ones (Rafal \& Henik, 1994; Tipper, Weaver, Jerreat, \& Burak, 1994). One particularly relevant study was reported by Schiller, Sandell, and Maunsell (1987). They showed that short-latency saccades under conditions containing a gap between fixation offset and target onset can still occur after frontal eye field ablation, but not after ablation of the superior colliculus (SC). Presumably, the SC is needed to produce the reflexive movements, whereas the frontal eye fields may be necessary for inhibiting such movements when they are not desired (as is consistent with the results of Guitton et al., 1985, discussed earlier). Other behavioral evidence and evidence from studies of patient populations suggests that there are important differences in the brain mechanisms involved in the production of endogenous and exogenous saccades (see Rafal \& Henik, 1994).

Given the different mechanisms that might underlie endogenous and exogenous saccades, a thorough understanding of the gap effect would not be complete without knowing whether the gap effect occurs for endogenous as well as exogenous saccades. For example, the gap effect may arise from processes involved in respond- ing to peripheral sensory targets, whether they be visual or auditory. In that case, a gap would not be expected to influence endogenous saccades. Nevertheless, despite its potential importance, no one has yet examined endogenous saccades under gap conditions.

Some researchers have examined the influence of a gap on antisaccades (saccades directed away from a suddenly appearing target). Reuter-Lorenz et al. (1991; Reuter-Lorenz et al., 1995) found that antisaccades were not initiated more quickly under gap than under no-gap conditions (but see the General Discussion). On the basis of those and other results, they concluded that the gap effect may arise from premotor processes in the SC. Antisaccades were presumably not affected by a gap, because they are less dependent on the colliculus, whereas peripheral auditory stimuli do yield a gap effect, because the colliculus is known to receive both auditory and visual inputs (see Fendrich et al., 1991).

On the surface, the absence of a gap effect for antisaccades suggests that the benefit of a gap may be limited to visually elicited saccades directed to a peripheral target. However, it is not clear whether the results from the antisaccade task can be extended to endogenous saccades in general. This is because antisaccades require active suppression of the reflex to look to the target onset, in addition to the production of the desired eye movement. Movements produced under those circumstances might be different from other endogenous saccades made in the absence of to-be-ignored target onsets. Thus, antisaccades may not provide a good test of the possibility that endogenous saccades are influenced by a gap. Our purpose in this study was to conduct such a test.

\section{EXPERIMENT 1}

We were interested in examining the possible presence of a gap effect for endogenous saccades in a situation in which subjects produced voluntary, centrally initiated, eye movements in response to a central signal. Subjects would still be required to produce an eye movement to one of several potential target locations, but they should not need to suppress a reflexive response to a visual signal located away from the target region. To accomplish this, we used the pitch of an auditory signal to designate the eye movement target. ${ }^{3}$ Note that this method differed from that used by Fendrich et al. (1991), who also used auditory stimuli. In their study, the spatial location of the auditory stimulus signaled the saccade target location.

\section{Method}

Subjects. Twenty Washington University undergraduates participated in a single 1-h session. They all had normal, uncorrected vision. They were paid $\$ 6$ for participation.

Apparatus and Procedure. The subjects were seated $38 \mathrm{~cm}$ in front of a video monitor in a dimly illuminated, sound-isolated room. They wore an infrared scleral reflectance eye movement monitor mounted to a spectacles frame (Applied Science Laboratories, Model 210) which monitored the movement of the right eye. The subjects' heads were kept steady by a chinrest. 


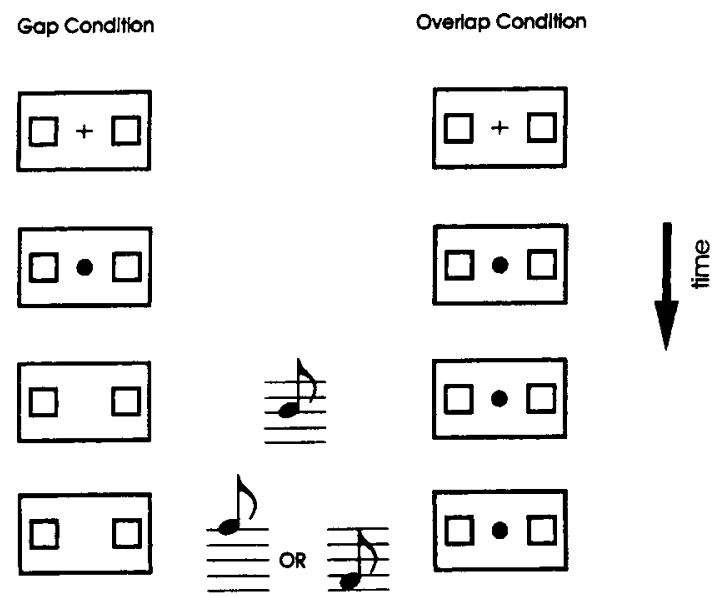

Figure 1. Sequence of events on a trial of Experiment 1. Two tones were presented on each trial: first, a warning tone of medium pitch, and then either a high- or a low-pitched tone. The pitch of the second tone indicated the direction of the required eye movement. On gap trials, the central fixation point was removed upon presentation of the first tone. See text for additional details.

The order of events on each trial is shown in Figure 1. At the beginning of each trial, a plus sign was presented in the center of the display $\left(0^{\circ}\right)$, and two boxes centered $7^{\circ}$ to the right and left of the plus sign appeared. These boxes served as targets and remained visible throughout the trial. The plus sign stayed visible for $300 \mathrm{msec}$ and then was replaced by a dot, which the subject was required to fixate upon. After an interval of $500 \mathrm{msec}$, two consecutive tones were presented from a single, small speaker centrally located beneath the video display. The first of these served as a warning tone and had a pitch of $800 \mathrm{~Hz}$ and a duration of $100 \mathrm{msec}$. The second tone, which was the target tone, also had a duration of $100 \mathrm{msec}$ and was presented $200 \mathrm{msec}$ after the onset of the warning tone. The pitch of the target tone (either 400 or $1400 \mathrm{~Hz}$ ) indicated the target to which the subject was required to look. The assignment of tones to targets was counterbalanced across subjects. The subjects were instructed to make a saccade as quickly as possible to the appropriate target box upon hearing the target tone.

There were two types of trials, defined by the presence of a gap between the offset of the fixation point and the onset of the target tone. On gap trials, the fixation dot disappeared at the onset of the warning tone. In this case, there was an interval of $200 \mathrm{msec}$ between the offset of fixation and the onset of the target tone. On overlap trials, the fixation dot remained visible throughout the trial.

Eye movement monitoring. At the beginning of each experimental session, the eye movement monitor was calibrated. In this procedure, the subjects fixated at each of five evenly spaced points across the display. Eye positions were determined by linear interpolation of the digitized signals from these points. Calibration was checked during each trial throughout the period before the target tone was presented. If the subjects were not fixating within $1.5^{\circ}$ of the fixation position, the calibration routine was repeated and the trial was rerun. Eye position was digitized and recorded at a rate of $1000 \mathrm{~Hz}$ for $600 \mathrm{msec}$ immediately after presentation of the target tone on each trial.

In order to identify saccades, the eye position signal was filtered and differentiated so that a smooth velocity record was obtained. The beginning of an eye movement was defined as the first moment in time when the velocity exceeded $10 \% \mathrm{sec}$ for at least $10 \mathrm{msec}$, with the constraint that velocity subsequently exceed $35 \%$ sec.

Design. The subjects served in one practice block of 20 trials, followed by five experimental blocks of 40 trials each. Half of the trials in each block were gap trials, and half were overlap trials. Left and right targets were equally likely.

\section{Results}

Reaction times. The saccade latencies were subjected to a 2 (gap condition) $\times 2$ (target location) analysis of variance (ANOVA). Mean latencies in each condition are shown in the triangles in Figure 2. As can be seen, subjects were faster to initiate eye movements to the target in the gap condition than in the overlap condition $[F(1,19)$ $=42.6, p<.0001]$. Seventeen of the 20 subjects showed the effect. There was no effect of target position $[F(1,19)$ $=3.0, p>.05]$, nor was there an interaction $[F(1,19)=$ $2.1, p>.15]$.

Errors. Error rates were also examined, to determine whether the shorter latencies in the gap condition could be due to a speed-accuracy tradeoff. The overall error rate was $13.6 \%$. An analysis revealed that the subjects did make more errors in the gap condition $(m=16.1 \%)$ than in the overlap condition $(m=11.2 \%)[F(1,19)=$ $13.2, p<.005]$.

\section{EXPERIMENT 1A}

Because the error rate in Experiment 1 was higher in the gap condition than in the overlap condition, it is possible that subjects in Experiment 1 were trading speed for accuracy, and that this might account for some of the observed reaction time differences. In order to rule out such an explanation, we replaced all subjects from Experiment 1 whose error rate exceeded $20 \%$ in any one condition. A total of 31 subjects were studied in order to obtain 20 that met this criterion. No other criteria were used to exclude subjects.

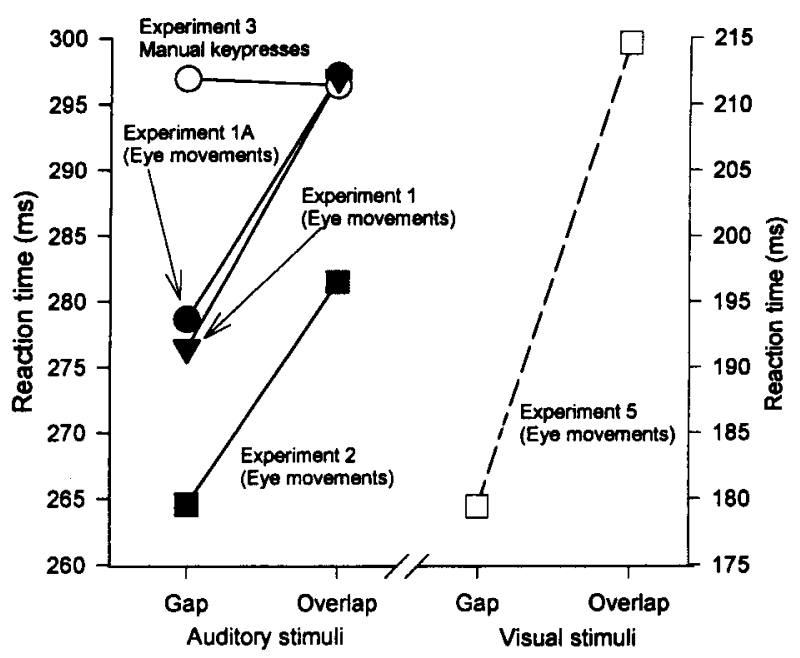

Figure 2. Mean reaction time for saccades from Experiments 1, 1A, 2, and 5, and manual keypresses from Experiment 3, separately for gap and overlap trials. The filled symbols represent endogenous saccades signaled by the pitch of a tone. The open squares represent exogenous saccades signaled by the onset of a peripheral visual target. 


\section{Results}

Reaction times. Saccade latencies are shown in the filled circles of Figure 2. As in Experiment 1, subjects were faster to move their eyes to the target in the gap condition than in the overlap condition $[F(1,19)=35.7$, $p<.0005]$. There was no effect of target position $[F(1,19)=1.5, p>.20]$, nor was there an interaction $[F(1,19)<1]$. Eighteen of the 20 subjects showed the effect.

We also conducted additional analyses to separately evaluate the two tone-target mappings (i.e., high or low tone assigned to either the left or the right target). This is because pitch is often regarded as increasing from left to right, as it does in musical instruments such as a piano. Thus, it would be important to examine our results separately for both "compatible" and "incompatible" stimulus-response mappings. The gap effect was the same for both tone-target mappings, as was evidenced by the absence of an interaction between condition and mapping $[F(1,18)<1]$. There was also no main effect of tone mapping $[F(1,18)=3.3, p>.05]$.

Errors. The overall error rate was $7.9 \%(8.6 \%$ for the gap condition, $7.2 \%$ in the overlap condition). Most importantly, an analysis of errors revealed no reliable effect of gap condition $[F(1,19)=3.6, p>.06]$ or target position $[F(1,19)=1.9, p>.1]$.

\section{Discussion}

In this experiment, the advance offset of the fixation point facilitated saccades whose direction was signaled by the pitch of an auditory signal. The gap effect for such movements occurred in the presence of a speed-accuracy tradeoff in Experiment 1, but the effect remained even when the error-prone subjects were replaced in Experiment 1A. Nevertheless, subjects were slightly, although not significantly, more error prone in the gap condition than in the overlap condition. This left open the possibility that at least some of the effect on latencies could be attributed to a speed-accuracy tradeoff. The next experiment was conducted to further discount this possibility.

\section{EXPERIMENT 2}

It was possible that the errors in the previous experiments could be attributed, at least in part, to the uncertainty that existed about details of the sequence of events on each trial. In particular, because gap and overlap trials were randomly mixed within a block, subjects could not predict whether the fixation point would or would not be disappearing on any given trial. Such uncertainty might have contributed to the high rate of erroneous responses. Thus, in Experiment 2, gap and overlap conditions were studied in separate blocks of trials. Although there was still uncertainty about which response would be called for, there was less uncertainty about the nature of the stimulus events that would occur on each trial.

\section{Method}

Subjects. Twenty-four Washington University undergraduates participated in a single 1 -h session. All had normal, uncorrected vision, and none had served previously. They were paid $\$ 6$ for participation.

Apparatus, Procedure, and Design. This experiment was identical to the previous experiment, the only exception being that gap and overlap trials were studied in separate blocks in the present experiment. The subjects served in four blocks of 36 trials under either the gap or the overlap condition, followed by four blocks of 36 trials in the other condition. Half of the subjects began with each condition. The target for each trial was randomly selected, with left and right targets equally likely within each block.

\section{Results}

Mean reaction times for the gap and overlap conditions are shown in the filled squares in Figure 2. As can be seen, subjects were faster to initiate saccades in the gap condition than in the overlap condition $[F(1,23)=$ $5.2, p<.05]$. The size of the effect $(16.9 \mathrm{msec})$ was very close to that in Experiment 1 A $(18.4 \mathrm{msec})$. There was no main effect of target $[F(1,23)<1]$, but the gap effect was somewhat larger for movements to the right $(21.6 \mathrm{msec})$ than for those to the left $[12.2 \mathrm{msec} ; F(1,23)=5.0$, $p<.05]$.

An analysis of errors revealed that subjects were not significantly more likely to make errors in the gap condition $(11.4 \%$ errors) than in the overlap condition $[9.4 \%$ errors; $F(1,23)=1.6, p>.2]$.

\section{Discussion}

In Experiments 1, 1A, and 2, subjects were faster to initiate saccades signaled by the pitch of the signal tone under gap conditions than under overlap conditions. In Experiment 1 , that effect was accompanied by significantly greater errors in the gap condition, but in Experiments $1 \mathrm{~A}$ and 2, there was no significant difference between the error rates in the two conditions. ${ }^{4}$ (In Experiment 1A, the error rate difference approached significance, but it did not in Experiment 2.) The presence of a gap effect for centrally produced endogenous saccades has important implications for current explanations of the mechanisms underlying the phenomenon. After addressing one potential limitation of the present experiment, we will address these issues in the General Discussion.

\section{EXPERIMENT 3}

There was one potentially serious limitation in the method of Experiments 1 and 2. We had assumed that the difference in latencies between gap and overlap conditions could be attributed specifically to the effect that the absence of a fixation point had on the oculomotor system. Nevertheless, it was possible that the latency difference that we observed was due to a more general warning signal effect. Indeed, the advance offset of fixation is capable of providing a generalized warning effect not specific to the oculomotor system (Kingstone \& Klein, 
1993a; Reuter-Lorenz et al., 1995). We attempted to equate such generalized warning effects between conditions by presenting the initial warning tone. Presumably the warning benefits of fixation offset in the gap condition would also be provided by the warning tone in the overlap condition. However, we included the warning tone in both gap and overlap conditions. As a result, it was possible that the general warning effect in the gap condition (with an offset plus a tone) exceeded the warning available in the overlap condition (tone only). If this were true, our latency difference might merely reflect an enhanced readiness to respond in general, and not the activity of processes specifically involved in making eye movements. In order to examine this possibility, we conducted the Experiment 3.

In Experiment 3, subjects received exactly the same stimuli under the same conditions as in Experiment 1. However, in the present experiment, subjects responded by pressing one of two response keys; they did not produce eye movements. If the shorter saccade latencies in the gap condition of Experiment 1 arose from a greater warning effect in that condition, the manual reaction times in Experiment 3 should also show the same pattern of results. However, if the results of Experiment 1 reflected oculomotor processes, the keypress latencies in Experiment 3 might be the same for both gap and overlap conditions.

\section{Method}

Subjects. Twenty Washington University undergraduates who had not served previously participated in a single $1-\mathrm{h}$ session. They all had normal, uncorrected vision. They were paid $\$ 6$.

Apparatus, Procedure, and Design. This experiment was identical to Experiment 1, except that the subjects were required to make finger presses in response to the target tone instead of eye movements. The subjects were instructed to remain fixated on the center dot throughout the trial. They responded by pressing one of two keys on a keyboard with either their right or left index finger. The correct key depended on the pitch of the target tone. Half the subjects made a right-hand response to a high tone, and half made a right-hand response to a low tone.

\section{Results}

The manual latencies for each subject were analyzed with a $2 \times 2$ (gap condition $\times$ target location) ANOVA. The mean latencies in each condition are shown in the open circles of Figure 2. The subjects were no faster to respond to target onset in the gap condition than in the overlap condition $[F(1,19)<1]$, and there was no effect of target position $[F(1,19)<1]$. The overall error rate, $5.7 \%$, did not depend on gap condition $[5.3 \%$ for gap, $6.3 \%$ for overlap; $F(1,19)=2, p>.15$ ].

\section{Discussion}

In Experiment 3, a gap between fixation offset and the onset of the target tone did not facilitate the production of manual keypress responses to the tone. This suggests that the benefit of the gap observed in Experiments 1 and 2 was not due to some generalized warning effect or to enhanced detection of the target. This is because any such effects would be expected to have influenced all responses, including the manual keypresses of the present experiment. Instead, the gap effect observed in Experiments 1 and 2 appears to have reflected processes specifically in the oculomotor system involved in the production of saccadic eye movements.

\section{EXPERIMENT 4}

There was one potential limitation of Experiment 3 as a control for general warning effects. The limitation stemmed from the possibility that the warning signal effect for manual responses might have had a time course that differed from that for ocular responses. Thus, although a 200-msec warning interval might facilitate the production of an eye movement, a 200 -msec warning might not have the same impact on the initiation of a manual response. If that were true, the absence of a gap effect on the keypress responses in Experiment 3 did not necessarily mean that there was not a general warning effect underlying the latency difference for the eye movements in Experiments 1 and 2. Experiment 4 was conducted to examine that possibility. In it, we studied manual keypress responses under gap and overlap conditions across a range of warning intervals.

\section{Method}

Subjects. Twenty Washington University undergraduates who had not served previously participated in a single 30 -min session. They were paid $\$ 5$.

Apparatus, Procedure, and Design. This experiment was similar to Experiment 3, except that we included warning intervals of 400 and $600 \mathrm{msec}$ in addition to the 200 -msec interval studied in Experiment 3 . After a practice block of 12 trials, each subject served in five blocks of 36 trials each. There were 12 unique trial types ( 3 warning intervals $\times$ gap/overlap $\times$ response left or right). Within a block, each of these occurred equally often, and in a random position.

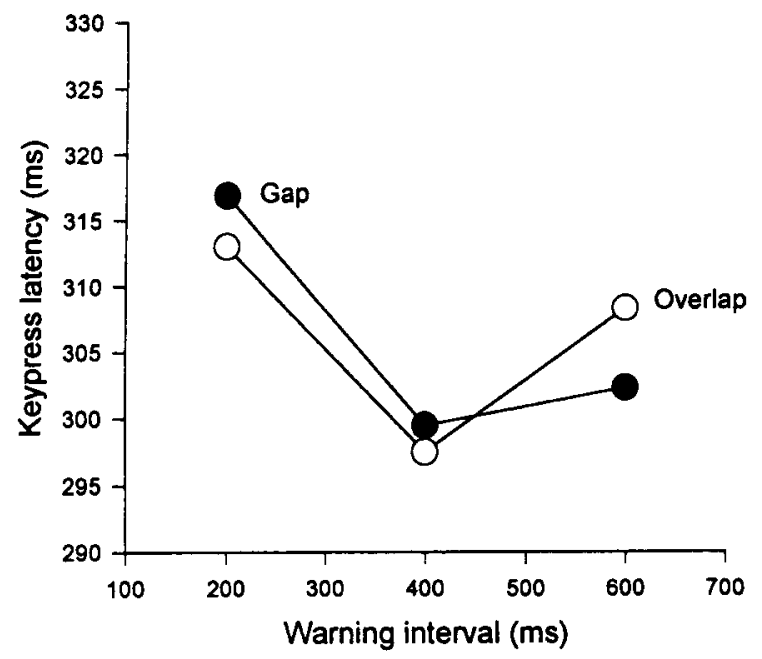

Figure 3. Mean reaction time for keypress responses from Experiment 4 . 


\section{Results and Discussion}

Mean keypress latencies are shown in Figure 3 separately for gap and overlap trials at each of the three warning intervals. As can be seen, there was no overall difference between gap and overlap conditions $[F(1,19)<1]$. There were reliable differences between the different warning intervals $[F(1,19)=6.6, p<.005]$, but warning interval did not interact with gap condition $[F(1,19)=1.1, p>.3]$.

These results served to further bolster our confidence in conclusions based on Experiment 3. In particular, the saccade latency differences observed in Experiments 1 and 2 did not appear to have arisen from general warning signal effects, which would have been expected to affect both saccade and manual response latencies, and instead could be assumed to reflect oculomotor phenomena.

\section{EXPERIMENT 5}

It was also of interest to determine whether the magnitude of the gap effect for endogenous, centrally produced saccades differs from that which would be obtained with the more typical suddenly appearing visual stimuli. An inspection of the literature suggests that the effect sizes that we observed (18.4 msec in Experiment 1A, $16.9 \mathrm{msec}$ in Experiment 2) were indeed smaller than those that had been obtained with visual onset stimuli. For example, Bekkering et al. (1996), using the same equipment as that in the present experiments, obtained a 45-msec gap effect for exogenous saccades in the most directly comparable conditions of their experiment. Despite the apparent difference in effect sizes, a direct comparison using a more similar method was desirable. To serve that purpose, we conducted Experiment 5.

In Experiment 5, we had subjects produce saccades under gap and overlap conditions under exactly the same circumstances as in Experiment $1 \mathrm{~A}$ with only one exception: here, instead of a high- or low-pitched tone to indicate the target at which to look, a small dot appeared inside the target box. Thus, this experiment involved suddenly appearing visual stimuli-the conditions under which the gap effect has traditionally been studied.

\section{Method}

Subjects. Ten Washington University undergraduates participated in a single 1-h session. They all had normal, uncorrected vision and had not served previously. They were paid $\$ 6$ for participation.

Apparatus, Procedure, and Design. This experiment was identical to Experiment $1 \mathrm{~A}$, except that the imperative stimulus was the onset of a dot in the center of the target box at which subjects were to look, instead of a tone as had been used in Experiment 1A. As in the earlier experiment, we also presented a warning tone in the present experiment $200 \mathrm{msec}$ before the presentation of the target. The subjects served in a total of eight blocks of 36 trials, with gap and overlap trials presented equally often and randomly within each block. The target for each trial was also randomly selected, with left and right targets equally likely within each condition.

\section{Results and Discussion}

Mean reaction times for the gap and overlap conditions are shown in the open squares of Figure 2. As can be seen, subjects were considerably faster to initiate saccades in the gap condition than in the overlap condition $[F(1,9)=112.4, p<.0001]$, with a mean effect size of $35.1 \mathrm{msec}$. The present effect, obtained with peripheral visual imperative stimuli, was significantly larger than the 18.4-msec effect obtained with central auditory stimuli in Experiment $1 \mathrm{~A}[F(1,28)=11.22, p<.005]$. Thus, the gap effect for reflexive, exogenous saccades appears to have been larger than the gap effect for endogenous saccades. The implications of these results will be discussed more fully in the General Discussion. Error rates were low, with subjects making slightly more errors $(5.7 \%)$ in the gap condition than in the overlap condition $[2.6 \% ; F(1,9)=6.5, p<.05]$.

\section{GENERAL DISCUSSION}

The present experiments demonstrate the presence of a gap effect for endogenous, centrally produced saccades. In Experiments 1, 1A, and 2, the saccade target was signaled by the pitch, not the location, of an auditory signal. The subsequent endogenous saccades were initiated more quickly following an offset at visual fixation. The results from Experiments 3 and 4, with manual keypresses, showed that the obtained effect could not be attributed to a generalized warning effect or to enhanced target detection. Instead, the fixation offset affected some processes involved in the production of the saccades. Experiment 5 showed that the benefit of fixation offset was greater for saccades produced to visual onset stimuli (i.e., exogenous saccades) than for those signaled by the pitch of a tone (i.e., endogenous).

\section{Presence of Gap Effect for Endogenous Saccades}

The existence of a gap effect for endogenous saccades provides new insight into the nature of the gap effect. It suggests that at least part of the gap effect is due to oculomotor processes involved in the production of a saccade, and not exclusively to processes involved in the perception of peripheral stimuli. This is because the effect was present under conditions in which all imperative stimuli were presented centrally.

These results are in apparent contrast to those of Reuter-Lorenz et al. (1991; Reuter-Lorenz et al., 1995). They showed that a gap did not reliably influence the latency of antisaccades (although a small nonsignificant effect in the correct direction was observed in both of their studies). However, we argued earlier that antisaccades might be special and might not be representative of endogenous saccades more generally, and the present results are consistent with that supposition.

\section{Comparison Between Endogenous and Exogenous Saccades}

An equally important result involves the comparison between the gap effects for endogenous and exogenous saccades. We found that the gap effect for endogenous saccades was significantly smaller than that for exoge- 
nous saccades. This result suggests that at least some of the gap effect may be attributed to relatively early processes, such as those involved in the processing of sensory signals, and not exclusively in later processes, such as those involved in the preparation and production of saccades. This is because the later movement-related processes would presumably be the same for endogenous and exogenous saccades.

These conclusions, however, should be made tentatively, because our results differ from those reported by Forbes and Klein (1996). Those researchers found equivalent gap effects for exogenous saccades and for endogenous saccades made in response to verbal commands. ${ }^{5}$ They concluded as a result that the gap effect must arise entirely from relatively late processes involved in the production of the saccades themselves, and not from earlier processes involved in evaluating sensory information. The ultimate resolution of this important issue may require further research.

\section{Role of the Superior Colliculus in the Gap Effect}

The existence of a gap effect for endogenous saccades provides some important new insights into the processes underlying the gap effect. As discussed earlier, a number of sources of evidence suggest that the gap effect is mediated by mechanisms in the SC. For example, Schiller et al. (1987) failed to find a gap effect after ablation of the SC. Reuter-Lorenz et al. (1991) found a gap effect only for target-directed saccades, not antisaccades or hand movements. And Fendrich et al. (1991) found a gap effect for saccades to acoustic targets. Because the SC is known to receive both auditory and visual inputs and is involved primarily in target-directed saccades, not antisaccades or hand movements, these results point to the $\mathrm{SC}$ as the locus of the gap effect. As suggested by ReuterLorenz et al. (1991; Reuter-Lorenz et al., 1995) and Fendrich et al. (1991), the gap effect may result from a release of inhibition in the SC that serves to inhibit eye movements during active fixation. Findings consistent with these possibilities have also been reported by Abrams and Dobkin (1994).

Nevertheless, there is also some evidence that the SC is also involved in aimed movements and in endogenous saccades. For example, Fries (1985) found projections from the arm representation area of motor and premotor cortex to the SC. And Werner (1993) has shown that neurons in the SC are active before and during arm movements to visual targets. Thus, it might be expected that disinhibition in the SC would also benefit hand movements. Indeed, Bekkering et al. (1996) have found a gap effect for aimed hand movements. Furthermore, there is also some reason to believe that even antisaccades, or endogenous saccades more generally, may benefit from a release of inhibition in the SC (Hughes, Reuter-Lorenz, Fendrich, \& Gazzaniga, 1992; Reuter-Lorenz et al., 1995). Such a possibility is consistent with the present findings, and with the trends reported by Reuter-Lorenz et al. (1991; Reuter-Lorenz et al., 1995) for antisaccades.

Of course, it is also possible that more than one process is affected by the advance offset of fixation. Bekkering et al. (1996) found that a gap reliably reduced latencies of aimed hand movements as well as eye movements, but the gap had a greater effect on the eye movement latencies. Thus, it may be that at least one component of the gap effect is unique to eye movements (perhaps influencing exogenous saccades only), whereas another component can affect responses more generally (such as aimed hand movements, endogenous saccades, and perhaps antisaccades).

\section{Conclusion}

The present results demonstrate that the gap effect is not limited to exogenous saccades, but instead can affect endogenous saccades also. Thus, the gap effect may be more general than was previously believed. To the extent that disinhibition in the SC may exert some influence on endogenous saccades, these results are consistent with explanations that attribute the effect to collicular mechanisms. It is our hope that further research on the phenomenon will shed more light on those mechanisms.

\section{REFERENCES}

Abrams, R. A., \& Dobkin, R. S. (1994). The gap effect and inhibition of return: Interactive effects on eye movement latencies. Experimental Brain Research, 98, 483-487.

Bekkering, H., Pratt, J., \& Abrams, R. A. (1996). The gap effect for eye and hand movements. Perception \& Psychophysics, 58, 628-635.

Cameron, E. L., \& LenNie, P. (1993). A speed/accuracy tradeoff in saccadic latency. Behavioral \& Brain Sciences, 16, 572-573.

Fendrich, R., Hughes, H. C., \& Reuter-Lorenz, P. A. (1991). Fixation-point offsets reduce the latency of saccades to acoustic targets. Perception \& Psychophysics, 50, 383-387.

Fischer, B., \& RAMSPERGER, E. (1984). Human express saccades: Extremely short reaction times of goal-directed eye movements. Experimental Brain Research, 57, 191-195.

Fischer, B., \& WEBER, H. (1993). Express saccades and visual attention. Behavioral \& Brain Sciences, 16, 553-610.

Forbes, K., \& KLEIN, R. M. (1996). The magnitude of the fixation offset effect with endogenously and exogenously controlled saccades. Journal of Cognitive Neuroscience, 8, 344-352.

FrIES, W. (1985). Inputs from the motor and premotor cortex to the superior colliculus in the rhesus monkey. Behavioural Brain Research, 18, 95-105.

Guitton, D., Buchtel, H. A., \& Douglas, R. M. (1985). Frontal lobe lesions in man cause difficulties in suppressing reflexive glances and in generating goal-directed saccades. Experimental Brain Research, $\mathbf{5 8 , 4 5 5 - 4 7 2 . ~}$

Hughes, H. C., Reuter-Lorenz, P. A., Fendrich, R., \& Gazzaniga, M.S. (1992). Bidirectional control of saccadic eye movements by the disconnected cerebral hemispheres. Experimental Brain Research, 91, 335-339

Kingstone, A., \& KLeIN, R. M. (1993a). Visual offsets facilitate saccadic latency: Does predisengagement of visuospatial attention mediate this gap effect? Journal of Experimental Psychology: Human Perception \& Performance, 19, 125 1-1265.

Kingstone, A., \& Klein, R. M. (1993b). What are human express saccades? Perception \& Psychophysics, 54, 260-273.

RAFAL, R., \& HeNiK, A. (1994). The neurology of inhibition: Integrat- 
ing controlled and automatic processes. In D. Dagenbach \& T. H. Carr (Eds.), Inhibitory processes in attention, memory, and language (pp. 1-51). San Diego: Academic Press.

Reuter-Lorenz, P. A., Hughes, H. C., \& Fendrich, R. (1991). The reduction of saccadic latency by prior offset of the fixation point: An analysis of the gap effect. Perception \& Psychophysics, 49, 167 175.

Reuter-Lorenz, P. A., Oonk, H. M., Barnes, L. L., \& Hughes, H. C. (1995). Effects of warning signals and fixation point offsets on the latencies of pro- vs. antisaccades: Implications for an interpretation of the gap effect. Experimental Brain Research, 103, 287-293.

Ross, S. M., \& Ross, L. E. (1981). Saccade latency and warning signals: Effects of auditory and visual stimulus onset and offset. Perception \& Psychophysics, 29, 429-437.

SASLOW, M. G. (1967). Latency for saccadic eye movement. Journal of the Optical Society of America, 57, 1030-1033.

SCHILler, P. H., SANDEll, J. H., \& Maunsell, J. H. R. (1987). The effect of frontal eye field and superior colliculus lesions on saccadic latencies in the rhesus monkey. Journal of Neurophysiology, 57, 1033-1049.

TipPer, S., WeAver, B., Jerreat, L. M., \& Burak, A. L. (1994). Objectbased and environment-based inhibition of return of visual attention. Journal of Experimental Psychology: Human Perception \& Performance, 20, 478-499.

WENBAN-Smith, M. G., \& Findlay, J. M. (1991). Express saccades: Is there a separate population in humans? Experimental Brain Research, 87, 218-222.

WERNER, W. (1993). Neurons in the primate superior colliculus are active before and during arm movements to visual targets. European Journal of Neuroscience, 5, 335-340.

\section{NOTES}

1. One exception to this is a paper by Forbes and Klein (1996) which appeared after the present article had been prepared. That article is discussed below.
2. It could be argued that this more natural scanning situation is more appropriate for studying the gap effect than the conditions that are typically used. This is because the typical situation studied (viz., the offset of fixation followed by onset of a target) is unlikely to occur naturally. However, it would be very natural if the disappearance of a fixated object facilitated the production of a centrally produced saccade which might be needed to continue some ongoing visual scanning. Thus, it might be even more beneficial for a gap to facilitate centrally produced saccades as opposed to stimulus-driven ones.

3. It is worth mentioning that an eye movement specified by the pitch of a tone is not purely endogenous in the sense that the subject must await an external signal before initiating the response. However, the spatial location of the saccade target is arbitrarily mapped to pitch, so a central decision must be made before the correct response can be produced. Consistent with current convention, we refer to this as an endogenous saccade.

4. After this article was prepared, we became aware of a manuscript reporting some similar results. Forbes and Klein (1996) found that subjects were approximately $44 \mathrm{msec}$ faster to initiate saccades to targets designated by verbal commands under gap as opposed to overlap conditions. In their study, however, subjects produced errors or anticipatory responses on $17.1 \%$ of the gap trials as opposed to $13.5 \%$ of the overlap trials. Thus, subjects appear to often be both faster and more error prone under gap conditions with these types of stimuli. (See also Cameron and Lennie, 1993, for a discussion of speed/accuracy tradeoffs in experiments involving the gap effect.)

5. It is worth noting that Forbes and Klein (1996) also observed significantly more errors in their gap condition than in their overlap condition for the endogenous saccades. Thus, it is possible that their endogenous gap effect is inflated in magnitude, contributing to their inability to detect a difference between endogenous and exogenous saccades.

(Manuscript received January 23, 1995; revision accepted for publication January $11,1997$. 